\title{
Ionic and dipolar solvation dynamics in liquid water ${ }^{+}$
}

\author{
NILASHIS NANDI, SRABANI ROY and BIMAN BAGCHI*† \\ Solid State and Structural Chemistry Unit, Indian Institute of Science, Bangalore 560012, \\ India \\ ${ }^{+}$Also at Jawaharlal Nehru Centre for Advanced Scientific Research, Bangalore and S N Bose \\ National Centre for Basic Sciences, Calcutta
}

\begin{abstract}
The solvation time correlation function for solvation in liquid water was measured recently. The solvation was found to be very fast, with a time constant equal to $55 \mathrm{fs}$. In this article we present theoretical studies on solvation dynamics of ionic and dipolar solutes in liquid water, based on the molecular hydrodynamic approach developed earlier. The molecular hydrodynamic theory can successfully predict the ultrafast dynamics of solvation in liquid water as observed from recent experiments. The present study also reveals some interesting aspects of dipolar solvation dynamics, which differs significantly from that of ionic solvation.
\end{abstract}

Keywords. Molecular hydrodynamic approach; dynamic mean spherical approximation; ionic solvation; dipolar solvation.

\section{Introduction}

The study of solvation dynamics of newly created charged species in dipolar liquids has been an area of intense research in the last decade (Bagchi 1989; Fleming and Wolynes 1990; Bagchi and Chandra 1991; Maroncelli 1993; Hynes 1994). This activity was fuelled by the availability of ultra-short laser pulses which made the study of solvation dynamics possible. In the first phase (roughly between 1986 and 1991) one could study solvation primarily in the slow liquids and attention was focussed on understanding the validity of continuum model (Bagchi 1989; Maroncelli et al 1989). In the second phase (roughly from 1991 to now), however, attention has shifted to the study of ultra-fast underdamped solvation in highly mobile liquids like water and acetonitrile (Rosenthal et al 1991; Roy and Bagchi 1993a; Jimenez et al 1994). In fact, a question that was repeatedly asked in the past, "How fast is the solvation in liquid water?" appears to have been provided an answer recently by Jimenez et al (1994), who measured this solvation for the first time. The solvation is extremely fast, with a time constant of about $55 \mathrm{fs}$.

Recently, we (Roy and Bagchi 1993b) have presented a theoretical calculation which predicted that solvation in real water could be significantly slower than that predicted in classical simulations (Maroncelli and Fleming 1988). This is because the classical simulations neglected polarizability and, therefore, missed much of the response from

\footnotetext{
+ Dedicated to Prof. C N R Rao on his 60th birthday

* For correspondence
} 
the $199 \mathrm{~cm}^{-1}$ intermolecular vibrational band. Thus, it was predicted that the time constant in real water could be around $70 \mathrm{fs}$ instead of the simulated value of $20 \mathrm{fs}$. Therefore, the experimental finding of the time constant of solvation as approximately equal to 55 fs was satisfactory.

However, the theory also predicted an oscillation at the intermediate time not observed in experiment. We, therefore, carried out a detailed study of the solvation dynamics with a view to understand the experimental result. This new calculation further differs in using more accurate dielectric relaxation data than were used previously.

The results are indeed interesting. It is found that if the high frequency modes of water are moderately damped, then an extremely good agreement with the experimental result is obtained. In addition, we present here the solvation dynamics of a point dipole in liquid water.

It is to be noted that precise dielectric relaxation data, which cover the whole time scale of the dynamical process under consideration, are necessary to compare the theoretical results with the experimental one. To deal with the complicated relaxation behaviour of an aqueous medium, it is thus certainly better to consider a suite of distinct relaxation processes with discrete relaxation times (Barthel et al 1990). So, we have used in the present study the experimental results from high frequency permittivity measurements (Barthel and Buchner 1991) with sufficiently large frequency coverage.

Water is a hydrogen-bonded liquid and shows special spectral features in the infrared region due to interaction induced dipoles (Madden and Impey 1986). To obtain the full information on the dynamics of the system, the inclusion of the far infrared range data is essential. Detailed information about the far infrared optical constants of liquid water is available (Hasted et al 1985) and we have exploited their results.

The solvation dynamics of ion or dipole can also be studied within the framework of mean spherical approximation (MSA). The mean spherical approximation has been extended to time domain to study the nonequilibrium solvation by Wolynes (1987). Here we have studied the time-dependent solvation of an ion and a dipole from MSA and compared with experimental and molecular hydrodynamic theory (MHT) calculation.

The organization of the rest of the paper is as follows. In $\S 2$ we have discussed the theoretical framework of the molecular hydrodynamic theory and the dynamic mean spherical approach, for ionic and dipolar solvation respectively. In $\S 3$ we have presented the results and their interpretation. Section 4 concludes with a brief summary of results.

\section{Theoretical discussion}

Here we briefly present the theoretical formulations for ionic and dipolar solvation by two currently popular theories, viz., the molecular hydrodynamic theory (Chandra and Bagchi 1989; Bagchi and Chandra 1991; Roy and Bagchi 1993) and the dynamic mean spherical approximation theory (DMSA) (Wolynes 1987; Nichols and Calef 1988; Rips et al 1988a). 


\subsection{Molecular hydrodynamic theory}

The progress of solvation of a nascent charge (ion or dipole) is usually described in terms of solvation time correlation function, $S(t)$, defined as

$$
S(t)=\frac{E_{\mathrm{sol}}(t)-E_{\mathrm{sol}}(\infty)}{E_{\mathrm{sol}}(0)-E_{\mathrm{sol}}(\infty)},
$$

where $E(t)$ is the time dependent solvation energy. In MHT, it is assumed to be given by the following expression

$$
E_{\mathrm{sol}}(t)=-\frac{1}{2} \int \mathrm{d} \mathbf{r} \mathbf{E}_{0}(\mathbf{r}) \cdot \mathbf{P}(\mathbf{r}, t) .
$$

Here, $\mathbf{E}_{0}(\mathbf{r})$ is the bare electric field of the charged solute at a position $\mathbf{r}$. The $\mathbf{P}(\mathbf{r}, t)$, is the space and time-dependent polarization of the solvent molecules and is the consequence of inhomogeneous density distribution around the solute molecule and. can be obtained from the MHT by solving the coupled conservation equations of number and momentum densities (Bagchi and Chandra 1991) and using the Fourier and Laplace transformations in space and time respectively. For computation of solvation energy the wave vector and frequency-dependent polarization is needed, and its longitudinal and transverse parts are given by (Roy and Bagchi 1993a)

$$
\mathbf{P}_{L}(\mathbf{k}, z)=\frac{\mathbf{P}_{L}(\mathbf{k}, t=0)}{z+\Sigma_{L}(k, z)},
$$

and

$$
\mathbf{P}_{T}(\mathbf{k}, z)=\frac{\mathbf{P}_{T}(\mathbf{k}, t=0)}{z+\Sigma_{T}(k, z)},
$$

where, $\Sigma_{L}$ and $\Sigma_{T}$ are longitudinal and transverse generalized rates, and are given by

$$
\begin{aligned}
& \Sigma_{L}(k, z)=\frac{2 f_{L}(k)}{\tau_{I}^{2}\left[z+\Gamma_{R}(k, z)\right]}+\frac{p k^{2} f_{L}(k)}{\tau_{I}^{2}\left[z+\Gamma_{T}(k, z)\right]}, \\
& \Sigma_{T}(k, z)=\frac{2 f_{T}(k)}{\tau_{I}^{2}\left[z+\Gamma_{R}(k, z)\right]}+\frac{p k^{2} f_{T}(k)}{\tau_{I}^{2}\left[z+\Gamma_{T}(k, z)\right]}
\end{aligned}
$$

Here, $\mathbf{k}$ is the Fourier variable conjugate to $\mathbf{r}$, and $\tau_{I}=\left(I / k_{B} T\right)^{1 / 2}$, denotes the time constant of free inertial motion of the solvent molecule. $p=I / m \sigma^{2}$ is the translational parameter and represents the relative weight of translational and rotational modes of the solvent. The static structural correlations of the pure solvent are expressed by (Bagchi and Chandra 1991)

$$
f_{L}(k)=1-\left(\frac{\rho_{0}}{4 \pi}\right) c(110 ; k),
$$

and

$$
f_{T}(k)=1+\left(\frac{\rho_{0}}{4 \pi}\right) c(111 ; k),
$$


where, $c(l l m ; k)$ denotes the $(l l m)$ component of the direct correlation function in the intermolecular frame, with $k$ parallel to the $z$ axis and related to the longitudinal and transverse part of wavevector dependent dielectric function by the relations (Bagchi and Chandra 1991)

$$
\varepsilon_{L}(k)=\left[1-3 Y\left(1-\frac{\rho_{0}}{4 \pi} c(110 ; k)\right)^{-1}\right]^{-1}
$$

and,

$$
\varepsilon_{T}(k)=\left[1+3 Y\left(1+\frac{\rho_{0}}{4 \pi} c(111 ; k)\right)^{-1}\right],
$$

where, the polarity parameter is given by $3 Y=(4 \pi / 3) \beta \mu^{2} \rho_{0}$ in terms of the dipole moment $\mu$ and the average number density, $\rho_{0}$ of water. $\beta=\left(k_{B} T\right)^{-1}\left(k_{B}\right.$ is the Boltzman constant and $T$ is the absolute temperature).

2.1a Ion solvation: Based on the above formulation, the time-dependent solvation energy of an ion, with charge $Q$, can be expressed in the frequency plane as follows

$$
E_{\text {sol }}(z)=-\frac{Q^{2}}{\pi \sigma^{2}} \int_{0}^{\infty} \mathrm{d} k\left(\frac{\sin k r_{c}}{k r_{c}}\right)^{2}\left[1-\frac{1}{\varepsilon_{L}(k)}\right] \frac{1}{z+\Sigma_{L}(k, z)} .
$$

Here $r_{c}$ is the distance of the closest approach between the solute ion and the solvent molecules. The computation of rotational and translational dissipative kernels are highly nontrivial, but for underdamped liquids (like water) a robust scheme of fully microscopic calculation of this kernel has been developed (Roy and Bagchi 1993). For ultrafast relaxation, relevant to underdamped solvation dynamics, the solvent response is expected to be fairly represented by its collective (that is, $k=0$ ) limit. The dissipative kerpel can then be determined using the following relation between $\Gamma_{R}(k=0, z)$ and the frequency-dependent dielectric function, $\varepsilon(z)$ (Roy and Bagchi 1993a),

$$
\frac{1}{\left.z+\Gamma_{R}(k=0, z)\right]}=\frac{z \tau_{I}^{2} \varepsilon_{0}\left[\varepsilon(z)-n^{2}\right]}{2 f_{L}(k=0) n^{2}\left[\varepsilon_{0}-\varepsilon(z)\right]},
$$

where, $\varepsilon_{0}$ and $n^{2}$ are the static and optical dielectric constants of liquid water. The dielectric relaxation experiments and far infrared FIR studies show complex frequency dependence of $\varepsilon(z)$ which may be represented as follows

$$
\varepsilon(z)=n^{2}+\sum_{i=1}^{2} \frac{\left(\varepsilon_{i}-\varepsilon_{i+1}\right)}{1+z \tau_{i}}+\sum_{j=1}^{3}\left(n_{j}^{2}-n_{j+1}^{2}\right)\left(1-z \Phi_{j}(z)\right),
$$

where $\tau_{i}, \varepsilon_{i}$ are the time constants and dielectric constants of the Debye relaxation, $n_{j}^{2}$ are the optical dielectric constants related to the high frequency librational modes and $\Phi_{j}(z)$ is the librational (or vibrational) moment correlation function corresponding to the $j$ th librational mode. This can be calculated using a model of underdamped harmonic oscillator with a librational frequency equal to $\Omega_{j}$ and a damping constant $\gamma_{j}$ (Hasted et al 1985). The time-dependent solvation energy can easily be obtained by Laplace inversion of the above equation in the time domain. 
2.1b Dipolar solvation: The dipolar field is anisotropic in nature (Gray and Gubbins 1984) and hence, unlike for an ionic field, we have to consider both the longitudinal and transverse components of the electric field of the solute dipole and also that of the space-and time-dependent polarization of the solvent (Bagchi and Chandra 1989; Chandra and Bagchi 1993). The time-dependent solvation energy of a dipole (dipole moment $\mu_{s}$ ) in water (dipole moment, $\mu_{w}$ ) in the frequency plane is given by

$$
\begin{gathered}
E_{\mathrm{sol}}(z)=-\frac{5 \mu_{s}^{2}}{2 \pi^{2} \sigma^{3}}\left[2 \int_{0}^{\infty} k^{2} \mathrm{~d} k\left(1-\frac{1}{\varepsilon_{L}(k)}\right)\left\{J_{1}^{2}\left[\frac{k(R+1)}{2}\right] /\left[\frac{k(R+1)}{2}\right]^{2}\right\}\right. \\
\frac{1}{z+\Sigma_{L}(k, z)}+\int_{0}^{\infty} k^{2} \mathrm{~d} k\left(\varepsilon_{T}(k)-1\right)\left\{J_{1}^{2}\left[\frac{k(R+1)}{2}\right] /\left[\frac{k(R+1)}{2}\right]^{2}\right\} \\
\left.\frac{1}{z+\Sigma_{T}(k, z)}\right]
\end{gathered}
$$

where $R$ is the solute-solvent size ratio (equal to $2 a / \sigma$, where $a$ is the solute radius), $J_{1}$ is the spherical Bessel function of first kind. The methods for obtaining the other necessary ingredients for calculation e.g. $\varepsilon(z), \Gamma_{R}, \Gamma_{T}$ etc. are similar to that described in $\$ 2.1$ a (for ionic solvation). The static transverse dielectric function $\varepsilon_{T}(k)$ has been calculated from the mean spherical approximation (MSA) model (Gray and Gubbins 1984). Since the large wave-vector process is more important in the case of dipolar solvation, MSA gives fairly accurate values for $\varepsilon_{T}(k)$. We have also compared the $\varepsilon_{T}(k)$ obtained from MSA with that of other theoretical approaches (Raineri and Friedman 1993) and good agreement has been observed. From the above equation the solvation time correlation function can again be obtained by numerical Laplace inversion.

\subsection{Dynamic mean spherical approximation (DMSA)}

Similar to MHT, DMSA also assumes the linear response of the solvent to the solute ion- (or solute dipole-) induced perturbation. The particular linearized theory used to analyse the non-equilibrium solvation is the mean spherical approximation (Wolynes 1987). The rotational dynamics of the solvent molecules is the only relaxation mechanism considered here. The effects of hydrodynamic and translational modes are neglected. The predictions of the DMSA model depend on $\varepsilon(z)$ (in addition, on the solute to solvent size ratio, for ionic solvation), which we can calculate from the method described earlier (Roy and Bagchi 1993a).

2.2a Ionic solvation: Within the framework of MSA, the ion solvation dynamics can be described as follows (Rips et al 1988a). Here, solutes are neutral hard spheres of radius $R$ and solvent (water) molecule has hard sphere radius $r_{w}$. The neutral solute is instantaneously ionized at time $t=0$, and its progress of solvation is then given by

$$
S(z)=\frac{[\chi(z)-\chi(0)]}{z[\chi(\infty)-\chi(0)]},
$$

where, $S(z)$ is the Laplace transform of the solvation time correlation function and 
$\chi(z)$ is the complex admittance of the solvent and is given by

$$
\chi(z)=\left\{\frac{1}{2 R} \frac{[1-(1 / \varepsilon(z))]}{(1+\Delta(z))}\right\} .
$$

Here, $\Delta(z)$ is the dynamic correction to the ionic radius, being the dynamic analogue of the Gurney co-sphere and can be approximated by

$$
\Delta(z)=\left(\frac{3 r_{w}}{R}\right)\left[108^{1 / 3}[\varepsilon(z)]^{1 / 6}-2\right]^{-1} .
$$

2.2b Dipolar solvation: MSA has been used to analyse the time-dependent solvation of a dipole in a dipolar solvent, for the special case, when the radius of the solvent molecules $r_{w}$ is equal to the solute radius $R$ (Rips et al 1988b). The solution of equalsized hard spheres with a permanent dipole moment (Wertheim 1971) has been used. The Laplace transform of solvation time correlation function is then given by

$$
S(z)=\frac{[h(\infty)-h(0)][h(0)-h(z)]}{z[h(0)-h(\infty)][h(z)+4]} .
$$

The $h(z)$ can be approximated by

$$
h(z)=4 \cdot 7622[\varepsilon(z)]^{1 / 6} .
$$

The numerical results obtained from the molecular hydrodynamic theory and mean spherical approximation are presented in the next section for both ionic-and dipolar solvation. For ionic solvation, the results are compared with current experimental results.

\section{Results and discussion}

In the present study we have used the experimental dielectric and FIR spectrum data (table 1) for obtaining the dissipative kernel and the interaction-induced dipoles of water are thus expected to contribute significantly to solvation dynamics. We have observed that the $199 \mathrm{~cm}^{-1}$ band has significant influence on initial decay and this band originates from interaction-induced dipoles as pointed out by Madden and Impey (1986). From the experimental information, the intermolecular motions have been related to the spectral features on the basis of semiquantitative arguments (Gulliot 1991). For instance, the band around $650 \mathrm{~cm}^{-1}$ is related with librational motions of the hydrogen-bond network. The $199 \mathrm{~cm}^{-1}$ band is correlated with $\mathrm{O} \ldots \mathrm{O}$ stretching mode of the $\mathrm{O}-\mathrm{H} \ldots \mathrm{O}$ units and the low frequency flexion of $\mathrm{O}-\mathrm{H} \ldots \mathrm{O}$ is assigned to the $65 \mathrm{~cm}^{-1}$ band.

The librational motions (torsional oscillations in the field of the solute and other solvent molecules) are particularly important for water, being a hydrogen-bonded solvent (Hynes 1994). The importance of translational vibrational modes in liquid water has already been pointed out in earlier studies (Roy and Bagchi 1993a, b). It is to be noted that the motions of the solvent molecules are described in terms of the molecular hydrodynamic equation with a dissipative kernel. The determination 
Table 1. The dielectric relaxation parameters of water.

(a) Time constants and dielectric constants of the Debye relaxation $\varepsilon_{1}=\varepsilon_{0}$, the static dielectric constant of the solvent; $\varepsilon_{3}=\varepsilon_{\infty}$, the infinite frequency dielectric constant obtained by fitting the low frequency relaxation to a Debye form.

\begin{tabular}{lllll}
\hline$\varepsilon_{1}$ & $\tau_{1}(\mathrm{ps})$ & $\varepsilon_{2}$ & $\tau_{2}(\mathrm{ps})$ & $\varepsilon_{3}$ \\
\hline 77.97 & 8.32 & 6.18 & 1.02 & 4.49 \\
\hline
\end{tabular}

Reference: Barthel and Buchner (1991)

(b) Frequency and dielectric constants for the high frequency librational modes $n_{3}^{2}=n^{2}$, the optical dielectric constant of the solvent.

\begin{tabular}{ccccccc}
\hline$n_{1}^{2}$ & $\Omega_{1}\left(\mathrm{~cm}^{-1}\right)$ & $n_{2}^{2}$ & $\Omega_{2}\left(\mathrm{~cm}^{-1}\right)$ & $n_{3}^{2}$ & $\Omega_{3}\left(\mathrm{~cm}^{-1}\right)$ & $n_{4}^{2}$ \\
\hline $4 \cdot 49$ & 50 & $4 \cdot 2$ & 199 & $2 \cdot 1$ & 650 & 1.77 \\
\hline
\end{tabular}

Reference: Hasted et al (1985)

of this kernel is made in terms of the dielectric function of water. The librational modes are treated as a set of harmonic oscillators that can mimic the effects of high frequency motions of water molecules. This implies that, in order to study the dynamics of the aqueous solvation, precise knowledge of the dynamics of these modes with their real physical characteristics (such as the mean frequency and the damping constant) is necessary (Ohmine et al 1988; Tanaka and Ohmine 1989; Ohmine and Tanaka 1990).

From recent studies on instantaneous normal mode analysis in water (Cho et al 1994) a more detailed picture of the nature of the high frequency modes of liquid water has emerged. A particularly interesting point is that the $199 \mathrm{~cm}^{-1}$ mode that is prominent in the quenched normal mode analysis (Ohmine and Tanaka 1993), almost disappears in the instantaneous normal mode calculations (Cho et al 1994). This is rather surprising and the reason for such behaviour is not well-understood (Ohmine, private communication). One possibility is that this mode is rather damped. The question, of course, remains why this mode is observed in dielectric relaxation or in Raman spectroscopy. One possibility is that local anharmonicity is important in the instantaneous normal mode but not in the macroscopic experiments. This point certainly deserves further study.

In this study we discovered that excellent agreement with experiments can be obtained if the $199 \mathrm{~cm}^{-1}$ mode is treated as overdamped. In the previous treatment this mode was treated as underdamped. While nearly perfect agreement was obtained with computer simulations, there was a significant oscillation in the theoretical result which was due to the assumed underdamping of the $199 \mathrm{~cm}^{-1}$ mode, which was not observed in the experiments.

In the case of dipolar solvation, the electric field has a shorter range than the ionic one and, consequently, the contributions of the nearest neighbours become more important than in the ionic case, and hence the contribution of the large wave-vector process becomes more pronouced. This in turn results in a slowing down of the initial decay (Rips et al 1988b). From figures 2 and 3 below, this feature has actually been observed from both MHT and DMSA theories in the present calculation. The present 


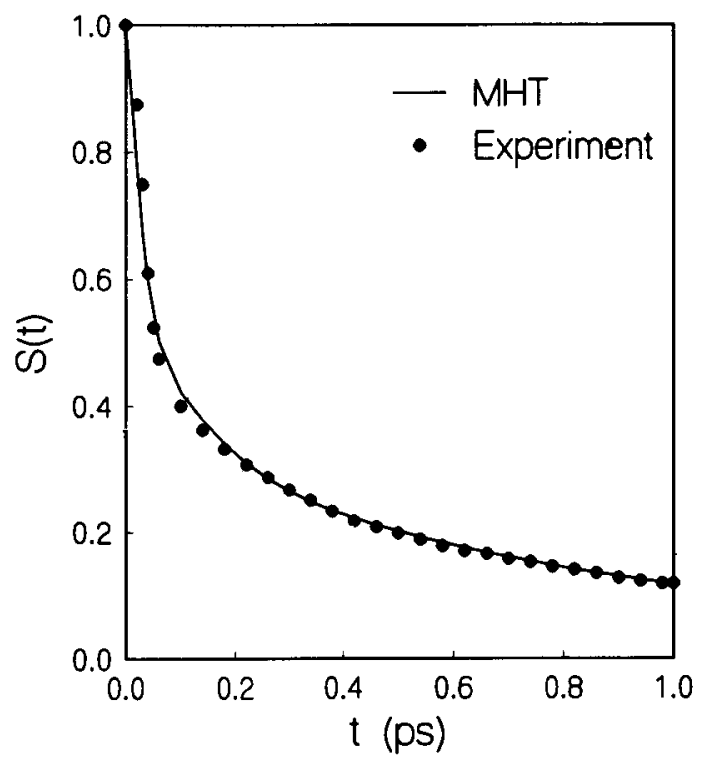

Figure 1. The calculated (from (11)) and experimental (Jimenez et al 1994) solvation time correlation function, $S(t)$, as a function of time for the solvation of an ionic solute (having size comparable to that of Coumarin-350 or C-350). The line represents the MHT prediction and the circles correspond to the experimental results. The damping constant $\gamma_{i}=x_{i} \Omega_{i}$, where, $x=2 \cdot 0,2 \cdot 0,0.5$ and $\Omega_{1}=50 \mathrm{~cm}^{-1}, \Omega_{2}=199 \mathrm{~cm}^{-1}, \Omega_{3}=650 \mathrm{~cm}^{-1}$. Other necessary parameters are given in the text.

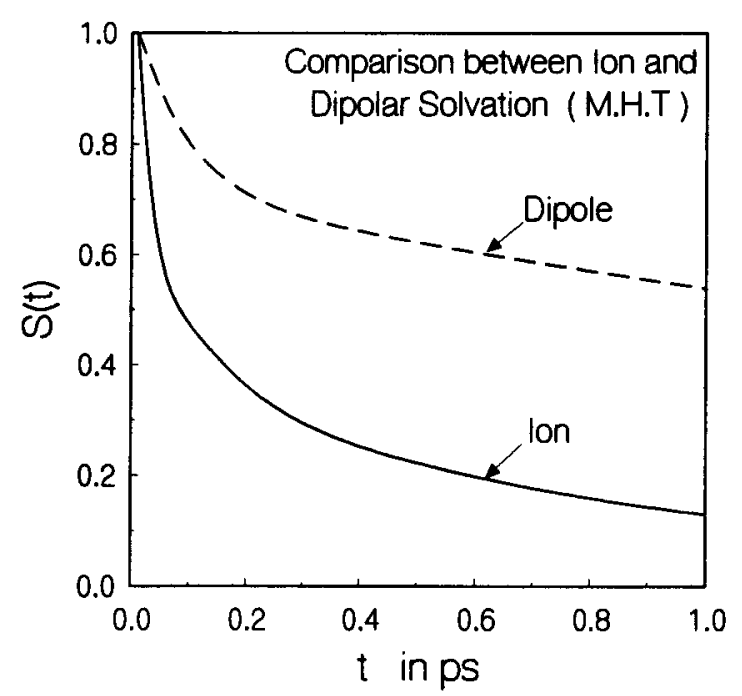

Figure 2. The calculated solvation time correlation function, $S(t)$, for an ionic and a dipolar solute (of size comparable to that of C-350 in each case) as a function of time, obtained from molecular hydrodynamic theory. The damping constants are the same as those given in figure 1. 


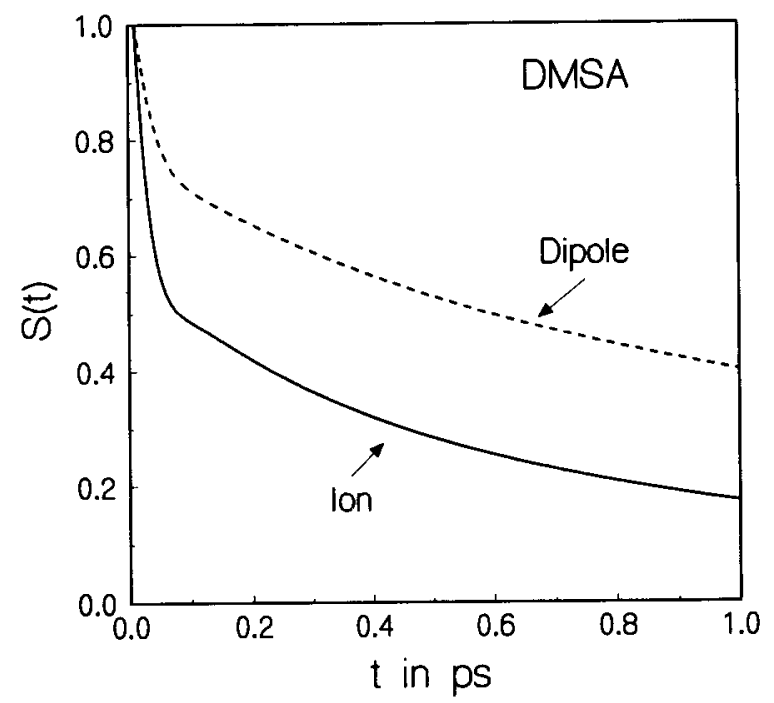

Figure 3. The calculated solvation time correlation function, $S(t)$, for an ionic and a dipolar solute as a function of time as obtained from dynamic mean spherical approximation (DMSA, (15) and (18)). Both solute and solvent are of equal size. The rest of the solvent parameters remain the same as in figure 1 .

DMSA calculation differs from the calculation oi Rips et al (1988) in using a better $\varepsilon(z)$ value (vide $\$ 2.1 \mathrm{a})$.

Figures 1,2 and 3 depict the solvation-time correlation function obtained from theoretical calculations, for both ionic and dipolar solvation. The ionic solute in the present study has a size comparable to that of Coumarin-343 (C-343) in order to compare our results with that of the experimental one (Jimenez et al 1994). In the case of dipolar solvation, the solvent and the solute dipoles are assumed to be of the same size.

The results of ion solvation are presented in figure 1. The agreement between MHT prediction and experimental situation is excellent. The calculated $S(t)$ has a pronounced bimodal character. The initial gaussian decay is extremely rapid, occurring at a time constant of 54 femtoseconds. This is in excellent agreement with experiment, which shows that a solvent response on a time scale of $55 \mathrm{fs}$ dominates the aqueous solvation dynamics and is attributed to the inertial (mainly librational) motions of the solvent molecules.

It may be noted that many real solute molecules have multipolar charge distribution and are not ionic in nature. Hence, a sum of dipolar, quadrupolar, octupolar etc. charge distributions is a better representation for such cases. The solvation dynamics are also expected to be different from that of ion solvation. A study similar to the present one may help in understanding the solvation dynamics of multipoles.

\section{Conclusions}

The present study shows that the initial part of the ultrafast solvation in liquid water can be described essentially in terms of librational motion of the solvent. The initial 
decay of the solvation time correlation function occurs with a time scale of $54 \mathrm{fs}$ which is in excellent agreement with experimental findings. The solvation dynamics of dipolar solutes have been observed to be much slower than in the ionic case because the nearest neighbour contribution becomes more pronounced.

\section{Acknowledgements}

NN thanks Dr A Chandra for valuable discussions. This work was supported by a grant from the Council of Scientific and Industrial Research, India.

\section{References}

Bagchi B 1989 Annu. Rev. Phys. Chem. 40 115, and related references therein

Bagchi B and Chandra A 1989 Chem. Phys. Lett. 155533

Bagchi B and Chandra A 1991 Adv. Chem. Phys. 801

Barthel J, Bachhuber K, Buchner R and Hetzenauer H 1990 Chem. Phys. Lett. 165369

Barthel J and Buchner R 1991 Pure Appl. Chem. 631473

Cho M, Fleming G R, Saito S, Ohmine I and Stratt R M 1994 J. Chem. Phys. 1006672

Chandra A and Bagchi B 1989 J. Phys. Chem. 936996

Chandra A and Bagchi B 1993 J. Chem. Phys. 99553

Fleming G R and Wolynes P G 1990 Phys. Today 4336

Gray C G and Gubbins K E 1984 Theory of molecular fluids (Oxford: Clarendon) vol. 1 Güillot B 1991 J. Chem. Phys. 951543

Hasted J B, Hussain S K, Frescura F A M and Birch R 1985 Chem. Phys. Lett. 118622.

Hynes J T 1994 Nature (London) 369471

Jimenez R, Fleming G R, Kumar P V and Maroncelli M 1994 Nature (London) 369461

Madden P A and Impey R W 1986 Chem. Phys. Lett. 123502

Maroncelli M 1993 J. Mol. Liq. 571

Maroncelli M and Fleming G R 1988 J. Chem. Phys. 895044

Maroncelli M, McInnis J and Fleming G R 1989 Science 2431674

Nichols A L III and Calef D F 1988 J. Chem. Phys. 891988

Ohmine I and Tanaka H $1990 \mathrm{~J}$. Chem. Phys. 938138

Ohmine I and Tanaka H 1993 Chem. Rev. 932545

Ohmine I, Tanaka H and Wolynes P G 1988 J. Chem. Phys. 895852

Raineri F O and Friedman H L 1993 J. Chem. Phys. 98, 8910

Rips I, Klafter J and Jortner J 1988a J. Chem. Phys. 883246

Rips I, Klafter J and Jortner J 1988b J. Chem. Phys. 894288

Rosenthal S J, Xie X, Du M and Fleming G R 1991 J. Chem. Phys. 954715

Roy S and Bagchi B 1993a Proc. Indian Acad. Sci. (Chem. Sci.) 105295

Roy S and Bagchi B 1993b J. Chem. Phys. 999938

Roy S and Bagchi B 1993c Ultrafast reaction dynamics and solvent effects. AIP Conference Proceedings, 298 (eds) Y Gauduel and P J Rossky (New York: Inst. Phys.)

Tanaka H and Ohmine I 1989 J. Chem. Phys. 916318

Wertheim M S 1971 J. Chem. Phys. 554291

Wolynes P G 1987 J. Chem. Phys. 865133 